\title{
Models for lifetime estimation: an overview with focus on applications to wind turbines
}

\author{
Thomas M. Welte $\cdot$ Kesheng Wang
}

Received: 16 January 2014/ Accepted: 14 February 2014/Published online: 15 March 2014

(C) Shanghai University and Springer-Verlag Berlin Heidelberg 2014

\begin{abstract}
This paper provides an overview of models and methods for estimation of lifetime of technical components. Although the focus in this paper is on wind turbine applications, the major content of the paper is of general nature. Thus, most of the paper content is also valid for lifetime models applied to other technical systems. The models presented and discussed in this paper are classified in different types of model classes. The main classification used in this paper divides the models in the following classes: physical models, stochastic models, data-driven models and artificial intelligence, and combined models. The paper provides an overview of different models for the different classes. Furthermore, advantages and disadvantages of the models are discussed, and the estimation of model parameters is briefly described. Finally, a number of literature examples are given in this paper, providing an overview of applications of different models on wind turbines.
\end{abstract}

Keywords Lifetime estimation - Model classification . Wind turbine

\section{Introduction}

An essential question for design and operation of technical components is their useful lives. The estimation of useful life is important for designers and manufacturers for design

T. M. Welte $(\bowtie)$

SINTEF Energy Research, 7465 Trondheim, Norway

e-mail: thomas.welte@sintef.no

\section{K. Wang}

Department of Production and Quality Engineering, Norwegian

University of Science and Technology, 7491 Trondheim,

Norway improvements and marketing purposes, as well as product users (customers, operators) for making decisions on which product to buy. Furthermore, the estimation of remaining useful life (RUL) after the product has been put into operation is important for making decisions on maintenance, repair, reinvestment and new investments. The RUL estimation is not only important for the users/operators but also for the manufacturers, especially in guarantee periods and when there are service agreements between users/ operators and manufacturers.

A large number of different models for estimation of lifetime and RUL exist. The purpose of this paper is to provide an overview of different models, classify the models in different groups and discuss advantages, disadvantages and methods for parameter estimation.

This paper provides an introduction to the topic of lifetime modeling by providing a brief overview of different types of models that are often applied. The paper may also serve as a good starting point for further reading. Readers that are familiar with only some models presented here may also contribute from this paper to become more familiar with models that they have not been applied before.

Lifetime models are often an integrated part of more complex models, for example, maintenance models and logistic models. The lifetime model is then sub-model in a more complex and larger main model. Consequently, the lifetime model is in this case an integrated part of the main model where the lifetime model processes data and generates results related to lifetime, and where the other parts of the main model process the results of the lifetime model further to results that are useful for making decisions on, for example, maintenance and logistics.

An important aspect of lifetime modeling is uncertainty. The lifetime of a technical component is an uncertain 
quantity that can never be predicted exactly. Thus, a lifetime model will result in an estimation rather than a certain and exact lifetime prediction. The overview in the paper includes different models and methods that can be used for handling and expressing uncertainty.

The paper is organized as follows. A classification of models for lifetime estimation is presented in Sect. 2. Typical model properties including their advantages and disadvantages and approaches for parameter estimation are discussed in Sect. 3. In Sect. 4, examples of different models are presented. In addition, references to publications presenting applications to wind turbines are given in Sect. 5. The paper is summarized in Sect. 6.

\section{Classification of models}

Models for lifetime estimation may be classified according to different aspects. The following main classification representing different main classes or main types of models is used in this paper. These model classes/types are physical models, stochastic models, data-driven models and artificial intelligence (AI), combined models.

Brief general descriptions of these types of models are given below. Further possibilities of model classification are discussed in Sect. 2.1.

\section{(i) Physical models}

Physical models (also called process-based models or mechanistic models [1]) are based on a physical mechanism (failure mechanism) or process (failure process) that finally leads to a failure of an item.

\section{(ii) Stochastic models}

Stochastic models are based on probability theory and statistical methods. If a model is "purely" stochastic it does not represent a physical process or mechanism, but is used to make a relation between model inputs and outputs using any mathematical equations or expressions that provide a good fit given the data [1].

\section{(iii) Data-driven models and AI}

Data-driven models (or data-based models) are based on methods to identify and abstract information and/or relationships from large sets of data [1]. Data-driven methods are in particular suitable for data from continuous monitoring. Many data-driven models belong to the field of AI.

AI is a field of research that studies and develops intelligent machines and software. A more formal definition of AI is "the field that studies the synthesis and analysis of computational agents act intelligently" where "an agent is something that acts in an environment" [2]. The agent is the modeling object, e.g., an animal, a machine, humans, companies, etc. The interest is "in what an agent does; that is, how it acts". Many different subfields, techniques and models are associated with AI (see Sect. 4.4). AI is based on general basic concepts such as search, learning, reasoning and others.

\section{(iv) Combination of models}

The combination of different models offers the possibility to establish new models. Both the combination of different types of models and the same type of model may improve a single model.

\subsection{Other possibilities of classification}

\subsubsection{Model quantity}

Another possibility of classification is according to the quantity that is modeled. These quantities that are often the prediction variables of lifetime models are the number of failures and failure time, and degradation. Thus, the models either belong to the class of failure (time) models or to the class of degradation models.

Failure models are used to model and predict failure events. The quantity usually modeled is the failure time and time to failure, the time between failures or the number of failures (in a given time interval or up to a given point in time), respectively. It might be more appropriate in some cases to use other measures of usage than time [3], e.g., revolutions (e.g., for rotating machines), number of operations (e.g., for switchgear), running times (e.g., for machines where it must be distinguished between operating and standstill) or load cycles (e.g., for structural components).

Degradation models are used to model and predict degradation of items. Degradation can be expressed and measured by a quantity which describes in a suitable way the changing of the technical condition or strength over time (or another measure of usage). This quantity is denoted degradation variable. Failure is assumed to occur when the value of a degradation variable crosses a failure level (threshold). In some applications, there may be more than one degradation variables [3].

\subsubsection{Model uncertainty}

One could also classify the models according to how they take into account uncertainty as follows:

(i) Deterministic: uncertainty is not taken into account.

(ii) Stochastic: uncertainty is modeled by probability theory.

(iii) Fuzzy: uncertainty is modeled by fuzzy logic theory. 


\subsubsection{Model generality}

A further possibility of model classification is the generality/universality of the model. Some models can be applied to all types of items and failure mechanisms, whereas others are specific models that only can be applied to the specific items and failure mechanisms they are designed for. Usually, specific models are more accurate than general models. However, the more specific and accurate a model is, the more complex it usually is.

\section{Model properties and parameter estimation}

\subsection{Physical models}

Since physical models are usually based on a physical mechanism (failure mechanism) or process (failure process), they are valid for all problems where the process/ mechanism leads to a failure. Sometimes, they are restricted to specific types of components. Typical for physical models is that the input parameters have a clear meaning and represent real (and often measurable) quantities or natural, physical or material constants. Thus, the models provide a clear understanding about the model input and output, resulting in a so-called white-box model. Therefore, physical models are appealing for those who wish to get better understanding of the mechanisms and processes leading to failure [1]. Physical models are in particular useful for design improvement.

In the first step, a physical model must be established if a good model is not available. This can be a challenging work and require good knowledge about the problem that is modeled. However, once a good model is available, it can be applied to all comparable problems where good estimates or measurements of the model input parameters are available. Since the processes in real world may be quite complicate and may be affected by many mechanisms and effects, one usually has not the possibility to take into account all of them. Thus, a physical model may be restricted to include the main mechanisms and main effects only.

Physical models are often empirical, which means they are based on observation or experiments. Physical models can basically be used for all kinds of predictions, both long-term and short-term, depending on what they are designed for.

\subsection{Stochastic models}

Most stochastic models are of general nature and can be applied for many different problems. An advantage of stochastic models applied to lifetime prediction is that both an estimate of the mean lifetime and various estimates of uncertainty can be established, such as variance of the lifetime, confidence intervals for parameters and predictions, etc.

Parameter estimation in stochastic modeling is based on the observation of the model output. Thus, observations of the model output, such as observations of lifetime or degradation, are usually collected as basis for parameter estimation. When possible, one should fit different stochastic models to the data and choose the model that gives the best prediction. Many techniques exist to choose the best model and check the goodness of fit (e.g., $p$-value, confidence intervals, comparison of the maximum likelihood values and various graphical methods such as probability plots).

As alternative to data collection, expert judgement can be used for parameter estimation. There exist different techniques for expert judgement (see Refs. [4, 5]).

Stochastic models can basically be used for both shortterm and long-term predictions. However, for lifetime prediction, they are mostly used to make medium and longterm predictions. Furthermore, they are often used in system modeling or as input in other models (such as maintenance and optimization models) where the main interest is in long-term averages (such as failure rates). They can also successfully be applied for comparing and explaining the lifetime influence of different designs or other factors either by looking on the results from different samples or by incorporating explanatory variables (see Sect. 4.3.4) in the model.

\subsection{Data-driven models and AI}

Data-driven techniques utilize monitored operational data related to system health. They can be beneficial when understanding of the first principles of system operation is not straightforward or when the system is so complex that developing an accurate alternative model is prohibitively expensive. An added value of data-driven techniques is their ability to transform high-dimensional noisy data into lower dimensional information useful for decision-making [6]. Furthermore, recent advances in sensor technology and refined simulation capabilities enable us to continuously monitor the health of operating components and manage the related large amount of reference data.

Many data-driven models can be classified as blackbox models because the relation of input and output variables and the model parameters is unclear in such types of models. Parameter estimation in black-box models is often based on learning and training. Thus, the models require data, and often data that covers a time period where a failure is observed, in order to make a prediction of the lifetime. Learning can be based on data 
from a situation identified as normal. Then, all situations that are different from the normal situation may be defined as abnormal and (potentially) erroneous. Such an approach is appealing for diagnostic applications, because the observation of failures is not required. However, this approach is not sufficient for making predictions of the remaining lifetime.

Data-driven models are mostly suitable for making short-term predictions when the component reaches the end of life and when a potential failure becomes apparent in monitoring data.

Since there are many models and methods in the field of AI that in addition often are quite different (also see examples of AI models in Sect. 4.4), it is difficult to make general statements about model properties and the ways of parameter estimation. Many models can be considered as black-box models. Some others however, as for example expert systems, are white-box models where the internal model logic is based on expert knowledge.

\section{Examples of models}

\subsection{Physical models}

When looking on wind turbine applications (also see Sect. 5), most models that have been applied in the class of physical models are models for fatigue lifetime prediction. Two of the basic material fatigue models are PalmgrenMiner rule (also simply called Miner's rule) and Paris' law, where the former can be classified as failure model, and the latter as degradation model.

\subsection{Fatigue and fatigue crack growth models}

\subsubsection{S-N-curves and Miner's rule}

$S$ - $N$-curves and the Miner's rule are failure models for fatigue life assessment of many types of materials.

If a material is subject to a sufficient number of stress cycles that are above the fatigue limit, a fatigue crack or damage will develop, finally leading to failure [7]. The higher the stress level, the less stress cycles are required until the item fails. This relation is illustrated by $S-N-$ curves (stress versus life curves) where $N$ is the number of cycles to failure. In a situation with constant amplitude loading, the number of stress cycles leading to failure, and the remaining lifetime, can be estimated by applying $S$ - $N$ curves. In a situation with variable amplitude loadings, the Miner's rule can be used together with $S$ - $N$-curves. More details about the approaches and their applications can be found in Ref. [7].

\subsubsection{Paris' law for fatigue crack growth}

Paris' law is a physical degradation model that describes the growing of a fatigue crack. The model can be used to predict the growing of a fatigue crack and estimate the RUL until fracture of the material. More details about the approach can be found in Ref. [7].

\subsection{Stochastic models}

\subsubsection{Stochastic failure rate models}

One of the main concepts in stochastic failure and reliability modeling is the failure rate (also called hazard rate). The constant failure rate, i.e., $\lambda(t)=\lambda$, is probably the most often applied failure and reliability model. Reasons for the popularity of using constant failure rates in lifetime and reliability analyses are as follows:

(i) The constant failure rate model is a simple model that has only one parameter.

(ii) The model parameter (the failure rate) has a clear meaning: expected number of failures within a time interval; the inverse of the failure rate is the mean time to failure (MTTF) or mean time between failures (MTBF).

(iii) Both data and expert judgment can easily be applied for estimating the failure rate. Methods exist for combination of data and expert judgement and updating of failure rate estimates; e.g., Bayesian methods [8].

(iv) Failure rates for different types of components can be found in databases and in the literature.

(v) Constant failure rates can often be applied in more advanced reliability and maintenance models, for example, in models for systems consisting of several items, Markov models or models for maintained items or systems (see Ref. [8]).

Obviously, the use of the very simple model of constant failure rates has restrictions. Constant failure rates are often not very realistic, because the failure rate for real components will often change during the lifetime, for example, due to aging or due to variable loadings. One of the distributions, which offers a time dependent failure rate function and which is often used in practical applications, is the Weibull distribution.

Different types of failure rate models exist for both repairable and non-repairable systems, for example:

(i) Stochastic failure rate models for non-repairable systems

- Failure time distributions (e.g., Weibull distribution) 
(ii) Stochastic failure rate models for repairable systems

- Renewal process

- Homogenous poisson process (HPP)

- Non-homogenous poisson process (NHPP)

- Trend renewal process

Failure time distributions are not further discussed here. It is referred to Refs. [3, 8] for further details. The stochastic processes for repairable systems listed above belong to the group of counting processes. A counting process is a general stochastic process for modeling of phenomena which may be described by the number of events (e.g., failures) that occur. The counting process is a stochastic process (i.e., collection of random variables) that represents the total number of events $n$ which occurred up to time $t$. More information about applications of different failure rate models and stochastic processes can be found in Refs. [3, 8-11].

The term failure rate is often ambiguously used for both non-repairable and repairable systems. Failure rate may refer in the former case to force of mortality (FOM) and in the latter case to rate of occurrence of failures (ROCOF). Whereas the FOM is a relative rate of hazard for a single item, the ROCOF is the absolute rate of change of an expected number of failures (see Ref. [12] for a more detailed discussion).

\subsubsection{Stochastic degradation models}

If degradation data are available, different stochastic degradation models can be used to model further degradation and predict the RUL. Some useful models are stochastic processes (e.g., Markov process, gamma process, Wiener process) or the general degradation path model presented in Ref. [3]. Further information about stochastic processes can be found in Ref. [9]. A good overview of application of the gamma process in lifetime and maintenance modeling is given in Ref. [13].

\subsubsection{Other approaches and models}

Other stochastic models that might be of interest are models for imperfect repair (see e.g., Ref. [8]), additive regression models of failure rate, relative risk regression, and frailty models (see Ref. [11]), and Bayesian belief networks, also called Bayesian networks (see Ref. [14]).

\subsubsection{Stochastic models with explanatory variables}

Stochastic models may include explanatory variables. Explanatory variables are also called covariates. An explanatory variable is a variable that may predict changes of the quantity of interest (i.e., the dependent variable, the lifetime or deterioration). In contrast to the parameters of the underlying stochastic model, explanatory variables have a clear/physical meaning, such as the parameters in physical models. When using stochastic models with explanatory variables, data must be collected on the value of the explanatory variables in addition to the value of the observation.

Examples of explanatory variables are environmental factors that may influence degradation and the lifetime, or accelerating factors such as higher stresses (often applied in accelerated life testing). Different types of technical designs can also be modeled by explanatory variables. Important factors for the wind industry one might to incorporate in the modeling are, wind speed, location of turbine or turbine concept/design.

\subsection{Data-driven models and AI}

It is difficult to classify models belonging to this model class. There exist a number of sub-fields/groups of models that are partly overlapping. AI includes sub-fields and groups such as machine learning, pattern recognition, computational intelligence (CI), expert systems, etc. Datadriven approaches can be divided into two categories: statistical techniques (regression methods, autoregressivemoving-average models, etc.) and CI techniques. The main focus in this section is on CI. However, other methods are briefly discussed in Sect. 4.4.2.

\subsubsection{Computational intelligence}

CI algorithms include artificial neural networks (ANN), evolutionary computation (EC), swarm intelligence (SI), artificial immune systems (AIS), and fuzzy systems (FS) [15]. CI-techniques have their origins in biological systems: "NNs model biological neural systems, EC model natural evolution (including genetic and behavioural evolution), SI models the social behaviour of organisms living in swarms or colonies, AIS models the human immune system, and FS originates from studies of how organisms interact with their environment" [15]. Detailed information about the different CI models and their applications can be found in Refs. $[15,16]$. Some CI models and their potential contributions and applications to RUL estimation are presented below.

\section{(i) Artificial neural networks}

With respect to $\mathrm{AI} / \mathrm{CI}$ techniques, the most commonly used prediction methods are based on ANN (see Refs. [1719]). For prognostic tasks, the most promising methods are back-propagation (BP) ANNs and recurrent neural networks (RNNs) (see Refs. [16, 20]).

For example, an ANN may be trained to predict a vibration value at one increment ahead of the previously 
sampled value. During training process, the previous sample is taken as training input, and the sample one time increment ahead is used as the output. The network is then trained by BP learning algorithm. During querying process of a trained ANN, we take the samples before the time interest as input. The output is then the predicted value for the next time interval.

ANNs are usually not directly used for lifetime estimation. However, ANN is a powerful tool for fault diagnosis, and in combination with other models (e.g., stochastic models or fuzzy logic), which may result in a good combined model (see Sect. 4.5) for lifetime estimation.

\section{(ii) Fuzzy logic systems}

An opportunity for increased transparency and openness of data-driven model is offered by fuzzy logic methods, which are increasingly proposed in modern diagnostic technologies. Based on the principles of Zadeh's fuzzy set theory, fuzzy logic provides a formal mathematical framework for dealing with the vagueness of everyday reasoning [21]. As opposed to binary reasoning based on ordinary set theory, within the fuzzy logic framework measurement uncertainty and estimation imprecision can be properly accommodated. Thus, fuzzy logic can be considered as an alternative to probability theory to describe uncertainty.

Yan et al. [22] and Zio and Maio [23] presented a similarity-based approach for prognostics of the RUL of a system. Data from failure dynamic scenarios of the system are used to create a library of reference trajectory patterns to failure. Given a failure scenario developing in the system, the remaining time before failure is predicted by comparing by the fuzzy similarity analysis of its evolution data to the reference trajectory patterns and aggregating their time to failure in a weighted sum which accounts for their similarity to the developing pattern. The prediction on the failure time is dynamically updated as time goes by, and measurements of signals representative of the system state are collected. The approach allows for the on-line estimation of the RUL.

\subsubsection{Other methods and models}

\section{(i) Statistical methods}

The most natural data-driven technique for RUL estimation is to fit a curve of the available data of the component degradation evolution using regression models and extrapolate the curve to the criteria (threshold) indicating failure. However, the available data history sometimes may be short and incomplete, so that extrapolation may lead to large errors. The same problem arises when employing autoregressive-moving-average (ARMA) models, although the method also can handle situations in which more runto-failure data are unavailable or insufficient [22].

\section{(ii) Pattern recognition}

Pattern recognition covers models and techniques to recognize patterns in data. Pattern recognition might be of interest for lifetime prediction in combination with casebased reasoning in order to extract an input case in form of a pattern that has been identified in connection with a failure. Then, the identification of the same or a similar pattern may provide a warning for a similar situation.

\section{(iii) Expert methods}

Case-based reasoning (CBR) is based on the principle to identify and solve problems based on experience from previous situations and similar problems. CBR can mean "adapting old solutions to meet new demands, using old cases to explain new situations, using old cases to critique new solutions, or reasoning from precedents to interpret a new situation (much as lawyers do) or create an equitable solution to a new problem (much as labor mediators do)" $[24,25]$. Obviously, data related to a potential (future) failure situation may be recognized from a similar case that has been observed before. The methods require the building of a data base with relevant cases.

In addition, rule-based expert systems [26] may be utilized for lifetime prediction. Expert knowledge and experience from observed failures may be utilized to establish suitable rules (e.g., if-then-rules).

\subsection{Combined models}

Some examples of models that may be classified as combined models are briefly described in the following.

\subsubsection{Physical models with uncertainty}

Deterministic physical models, for example, can be transferred into models that take into account uncertainty by modeling one or several model parameters as stochastic or fuzzy variables.

The stress-strength approach, also called load-strength approach [27], is based on the general principle that failure occurs when the load or stress $l$ exceeds the strength $s$ of the item. Thus, the model is based on the simple deterministic relation that a failure occurs when $l>s$. For most items neither load nor strength is deterministic, but distributed statistically. This means that the values of both the load and the strength are uncertain, and this uncertainty is modeled by assuming that load and strength are modeled as stochastic variables. 


\subsubsection{Finite element methods (FEM)}

Modern computer technology allows reducing complicate problems to simple computational steps that can easily be solved. These steps are usually based on one or several simple laws or computational rules, such as physical models or stochastic models. A repetition of the simple steps, following by an aggregation and combination of the calculation results, makes the solution of larger problems manageable. FEM, for example, utilizes this principle.

\subsubsection{Neuro-fuzzy systems}

Hybrid CI models are able to integrate ANN models and fuzzy logic system models to make a better performance [28].

\section{Applications to wind turbines}

This section provides a short overview of applications to wind turbines (see Table 1). The aim of this section is not to provide a complete literature review, but a brief overview of selected applications presented in the literature.

\subsection{Physical models}

Paris' law, the $S$ - $N$-curve approach and related approaches have frequently been applied to fatigue life analysis of wind turbine rotor blades. A comprehensive overview can be found in Refs. [29, 30]. Other examples with applications to wind turbines are presented in Refs. [31-36].

\subsection{Stochastic models}

The Weibull distribution has been used in Refs. [33, 37, 38] to model failures of wind turbines or different turbine components.

Table 1 Applications to wind turbines

\begin{tabular}{|c|c|c|}
\hline Physical models & $\begin{array}{l}\text { Stochastic } \\
\text { models }\end{array}$ & $\begin{array}{l}\text { Data-driven } \\
\text { models/AI }\end{array}$ \\
\hline $\begin{array}{l}\text { Paris' law, } S \text { - } N \text {-curves } \\
\text { and models related to } \\
\text { fracture mechanics } \\
\text { [29-36] }\end{array}$ & $\begin{array}{l}\text { Weibull distribution } \\
{[33,37,38]} \\
\text { Poisson process } \\
{[37,39-41]} \\
\text { Power law process } \\
{[42-45]} \\
\text { Gamma/Markov } \\
\text { process } \\
\text { [46-48] } \\
\text { Bayesian network } \\
{[31]}\end{array}$ & $\begin{array}{l}\text { ANN, fuzzy } \\
\text { sets } \\
{[49]}\end{array}$ \\
\hline
\end{tabular}

In Refs. $[37,39,40]$, the Poisson process is applied to carry out analyses of failure data available from existing wind power plants. Rademakers et al. [41] used the homogenous Poisson process to model gearbox bearing failures. The power low process has been used in Refs. [42-45] to model failures of wind turbines and different wind turbine components. The data for parameter estimation in these models are taken from different European data bases (Danish and German data sources such as Windstats and LWK).

The Markov and Gamma process is applied in Ref. [46] to model gearbox failure. In Ref. [47], the Markov process is used to model cracks and sudden failure of wind turbine blades. In Ref. [48], a general Markov process model is presented.

The use of Bayesian networks in wind turbine reliability modeling is described in Ref. [31].

\subsection{Data-driven models and AI}

Applications of data-driven models and AI models to wind turbine lifetime prediction are difficult to find. As already stated in Sect. 3.3, these models are mostly suitable for making short-term predictions. Thus, they are often applied to fault detection where the model provides a warning a short time period before the failure actually occurs, without providing an estimation of the remaining time to failure. Case studies using real SCADA and condition monitoring data from wind turbines show that the models presented are capable to provide warnings of potential failures either hours [50-52] or days [53] before they actually occur. Some of the models and methods that have been applied in these studies are ANNs, support vector machines, principle component analysis, auto-associative neural networks and self-organizing feature maps.

A model for prediction of the RUL can be found in Ref. [49]. The paper presents a model where an ANN is used for measurement value prediction and the residuals between predicted and observed values are the basis for comparing data related to observed failures with a current fault evolution. The comparison of the residuals in combination with fuzzy sets results in a fuzzy remaining time to failure prediction.

\section{Summary}

This paper shows that there are many different models that may be used for lifetime estimation. All models can be classified in a large multi-dimensional space of model classes representing different aspects, e.g., as suggested in this paper: main class/type of model, the quantity that is modeled, the way uncertainty that is handled and the model generality. 
In addition, this paper discusses different model properties and parameter estimations. Based on the information presented in the paper, it should be possible to select one or several models, or a class of models, that may be appropriate for lifetime estimation given a specific application, given the data and knowledge available and given the preferences regarding handling of uncertainty, prediction interval (long- or short term) and generality.

Of course, it is not possible to list all available models and groups of models in such a paper. Thus, the descriptions are restricted to a general and overview level. Nevertheless, the paper may be a useful starting point for getting a general overview of the specific field of lifetime modeling. Finally, the paper may be helpful in selecting lifetime models, especially for readers who are not familiar with the specific field.

Acknowledgement This paper is based on the results from the research projects Windsense - Add-on instrumentation system for wind turbines (Grant No. 217607) and NOWITECH - The Norwegian Research Centre for Offshore Wind Technology (Grant No. 193823). The financial support by the project participants, the research centre members and the Research Council of Norway is gratefully acknowledged.

\section{References}

1. Loucks DP, van Beek E (2005) Water resources systems planning and management: an introduction to methods, models and applications, Chap. 6 "Data-based models". United Nations Educational, Scientific and Cultural Organization, Paris

2. Poole DL, Mackworth AK (2010) Artificial intelligence: foundations of computational agents. Cambridge University Press, Cambridge

3. Meeker WQ, Escobar LA (1998) Statistical methods for reliability data. Wiley, New York

4. Cooke RM (1992) Experts in uncertainty: opinion and subjective probability in science. Oxford University press, New York

5. Meyer MA, Booker JM (2001) Eliciting and analyzing expert judgment: a practical guide. Academic Press, London

6. Dragomir OE, Gouriveau R, Zerhouni N et al (2007) Framework for a distributed and hybrid prognostic system. In: The 4th IFAC conference on management and control of production and logistics

7. Dowling NE (1999) Mechanical behavior of materials: engineering methods for deformation, fracture and fatigue. Prentice Hall, Upper Saddle River

8. Rausand M, Høyland A (2004) System reliability theory: models, statistical methods, and applications, 2nd edn. Wiley, Hoboken

9. Ross SM (1996) Stochastic processes, 2nd edn. Wiley, New York

10. Lindqvist BH, Elvebakk G, Heggland K (2003) The trendrenewal process for statistical analysis of repairable systems. Technometrics 45:31-44

11. Aalen OO, Borgan $\varnothing$, Gjessing HK (2008) Survival and event history analysis: a process point of view. Springer, New York

12. Ascher H, Feingold H (1984) Repairable systems reliability: modeling, inference, misconceptions and their causes. CRC Press/Marcel Dekker, New York

13. van Noortwijk JM (2009) A survey of the application of gamma processes in maintenance. Reliab Eng Syst Saf 94:2-21
14. Jensen FV (2001) Bayesian networks and decision graphs. Springer, New York

15. Engelbrecht AP (2007) Computational intelligence: an introduction, vol 2. Wiley, Chichester

16. Wang K (2005) Applied computational intelligence in intelligent manufacturing systems. Advanced Knowledge International, Adelaide

17. Peel L (2008) Data driven prognostics using a Kalman filter ensemble of neural network models. In: International conference on prognostics and health management

18. Barlett EB, Uhrig RE (1992) Nuclear power plant status diagnostics using an artificial neural network. Nucl Technol 97:272-281

19. Santosh TV, Srivastava A, Sanyasi Rao VVS et al (2009) Diagnostic system for identification of accident scenarios in nuclear power plants using artificial neural networks. Reliab Eng Syst Saf 94:759-762

20. Campolucci P, Uncini A, Piazza F et al (1999) On-line learning algorithms of locally recurrent neural networks. IEEE Trans Neural Netw 10:253-271

21. Zadeh L (1965) Fuzzy sets. Inform Control 8:338-353

22. Yan J, Koç M, Lee J (2004) A prognostic algorithm for machine performance assessment and its application. Prod Plan Control 15:796-801

23. Zio E, Maio FD (2010) A data-driven fuzzy approach for predicting the remaining useful life in dynamic failure scenarios of a nuclear system. Reliab Eng Syst Saf 95:49-57

24. Kolodner JL (1992) An introduction to case-based reasoning. Artif Intell Rev 6:3-34

25. Kolodner JL (1993) Case-based reasoning. Morgan Kaufmann, San Mateo

26. Nilsson NJ (1998) Artificial intelligence: a new synthesis. Morgan Kaufmann, San Francisco

27. O'Connor PDT, Newton D, Bromley R (2002) Practical reliability engineering, 4th edn. Wiley, Chichester

28. Wang WQ, Goldnaraghi MF, Ismail F (2004) Prognosis of machine health condition using neuro-fuzzy systems. Mech Syst Signal Process 18:813-831

29. Sutherland HJ (1999) On the fatigue analysis of wind turbines. Report No. SAND99-0089. Sandia National Laboratories, Albuquerque

30. Nijssen RPL (2007) Fatigue life prediction and strength degradation of wind turbine rotor blade composites. Dissertation, Delft University

31. Nielsen JJ, Sørensen JD (2010) Bayesian networks as a decision tool for O\&M of offshore wind turbines. In: Proceedings of the 5th international ASRANet conference

32. Nielsen JJ, Sørensen JD (2011) On risk-based operation and maintenance of offshore wind turbine components. Reliab Eng Syst Saf 96:218-229

33. Gray CS, Watson SJ (2009) Physics of failure approach to wind turbine condition based maintenance. Wind Energy 13:395-405

34. Marin JC, Barroso A, Paris F et al (2007) Study of damage and repair of blades of a $300 \mathrm{~kW}$ wind turbine. Energy 33:1068-1083

35. Ronold KO, Wedel-Heinen J, Christensen CJ (1999) Reliabilitybased fatigue design of wind-turbine rotor blades. Eng Struct 21:1101-1114

36. Sutherland HJ, Mandell JF (1996) Application of the U.S. high cycle fatigue data base to wind turbine blade lifetime predictions. In: Proceedings of energy week 1996. ASME (American Society of Mechanical Engineers), Calgary

37. Guo H, Watson S, Tavner P et al (2009) Reliability analysis for wind turbines with incomplete failure data collected from after the date of initial installation. Reliab Eng Syst Saf 94:1057-1063

38. Andrawus JA, Watson J, Kishk M (2007) Modelling system failures to optimise wind turbine maintenance. Wind Eng 31:503-522 
39. Spinato F, Tavner PJ, van Bussel GJW et al (2008) Reliability of wind turbine subassemblies. IET Renew Power Gener 3:387-401

40. Tavner PJ, van Bussel GJW, Spinato F (2006) Machine and converter reliabilities in wind turbines. In: Proceedings of the IET 3rd international conference on power electronics, machine and drives, 4-6 April 2006, Dublin

41. Rademakers LWMM, Braam H, Verbruggen TW (2003) R\&D needs for O\&M of wind turbines. In: European wind energy conference 2003, 16-19 June 2003, Madrid

42. Coolen FPA, Spinato F, Venkat D (2008) On modelling of grouped reliability data for wind turbines. IMA J Manag Math 21:363-372

43. Tavner PJ, Ciang J, Spinato F (2005) Improving the reliability of wind turbine generation and its impact on overall distribution network reliability. In: Proceedings of the 18th conference on electricity distribution (CIRED 2005), 6-9 June 2005, Torino

44. Tavner P, Edwards C, Brinkman A et al (2006) Influence of wind speed on wind turbine reliability. Wind Eng 30:55-72

45. Tavner PJ, Xiang J, Spinato F (2007) Reliability analysis for wind turbines. Wind Energy 10:1-18

46. Hameed Z, Vatn J (2012) State based models applied to offshore wind turbine maintenance and renewal. In: Berenguer C, Grall A, Soares CG (eds) Advances in safety, reliability and risk management, proceedings of the European safety and reliability conference, ESREL 2011. CRC Press/Balkema, Leiden, pp 989-996

47. Besnard F, Bertling L (2010) An approach for condition-based maintenance optimization applied to wind turbine blades. IEEE Trans Sustain Energy 1:77-83

48. Byon E, Ding Y (2010) Season-dependent condition-based maintenance for a wind turbine using a partially observed Markov decision process. IEEE Trans Power Syst 25:1823-1834

49. Garcia MC, Sanz-Bobi MA, del Pico J (2006) SIMAP: intelligent system for predictive maintenance application to the health condition of a wind turbine gearbox. Comput Ind 57:552-568

50. Kusiak A, Verma A (2012) Analyzing bearing faults in wind turbines: a data-mining approach. Renew Energy 48:110-116

51. Kusiak A, Li W (2011) The prediction and diagnosis of wind turbine faults. Renew Energy 36:16-23

52. Mesquita Brandão RF, Beleza Carvalho JA, Maciel Barbosa FP (2012) Forecast of faults in a wind turbine gearbox. In: Elektro 2012, pp 170-173

53. Kim K, Parthasarathy G, Uluyol O et al (2011) Use of SCADA data for failure detection in wind turbines. In: Energy sustainability conference and fuel cell conference, 7-10 August 2011, Washington, DC 